\title{
Side-Chain Liquid Crystal Block Copolymers with Well-Defined Structures Prepared by Living Anionic Polymerization I. Thermotropic Phase Behavior and Structures of Liquid Crystal Segment in Lamellar Type of Microphase Domain
}

\author{
Masayuki Yamada, Tsukasa Iguchi, Akira Hirao, \\ Seiichi NaKahama, and Junji Watanabe ${ }^{\dagger}$ \\ Department of Polymer Chemistry, Tokyo Institute of Technology, \\ Ookayama, Meguro-ku, Tokyo 152, Japan
}

(Received May 9, 1997)

\begin{abstract}
We have synthesized the A-B block copolymer with polystyrene as A segment and side-chain liquid crystal (LC) polymer as B segment by a sequential living polymerization. The copolymers were successfully prepared with quantitative yields, possessing the predictable $M_{n}$ values, compositions, and narrow molecular weight distributions. The thermotropic phase behavior and structures were examined for nine copolymers with the fraction of segments around $50 \mathrm{w} \%$ and with the various molecular weights ranged from 8000 to 34000 . All the polymers exhibit the well-defined lamellar type of segregation and the side-chain LC segment in the microdomain forms crystal, smectic A $\left(S_{\mathrm{A}}\right)$ and isotropic phases with increasing temperature. The lamellar segregation is maintained over the whole temperature region from crystal to isotropic phases, and the crystal and $S_{\mathrm{A}}$ structures are formed with a preferential orientation to the microdomain interface such that the mesogenic side-chain groups lie parallel to the interface. The lamellar thickness depends on the $S_{\mathrm{A}}$ temperatures. It gradually decreases from the value of crystal phase to that of isotropic phase. The reduction is around $20-25 \%$, which can be explained in terms of the change in main-chain conformation of the LC block.

KEY WORDS Block Copolymer / Liquid Crystal / Smectic Phase / Microphase Separation / Lamellar Structure / Living Polymerization /
\end{abstract}

Liquid crystal (LC) polymers with mesogenic groups in the side chains (side-chain LC polymers) have been extensively studied. ${ }^{1,2}$ More recently, the research interests have focused on a role of the main-chain backbone on liquid crystal structures and properties. The various parameters with respect to the main-chain backbone such as a degree of polymerization, molecular weight distribution, tacticity, and monomer unit distribution in copolymers can be considered. An essential breakthrough can be performed by controlled polymerization such as group-transfer polymerization and living polymerization, since these permit the control of several parameters given above because of the synthesis of welldefined structures. ${ }^{3-8}$

In the previous paper, ${ }^{9}$ we succeeded to prepare a series of side-chain LC polymers with a well-controlled structure, by living anionic polymerization. Polymerization was attempted at an elevated temperature of $-40^{\circ} \mathrm{C}$ since precipitation occurs during the polymerization at a usually applied temperature of $-78^{\circ} \mathrm{C}$. Irrespective of this unusual condition for the living anionic polymerization, the polymers were obtained with unimodal distribution of less than 1.1 when the molecular weights are less than 12000. Their thermotropic phase behavior and structures were examined as a function of the molecular weight.

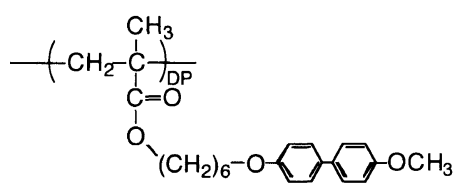

Poly(1)

† To whom correspondence should be addressed.
One of the most attractive features of living polymerization is a feasibility of synthesizing block copolymers with well-defined structures. In the copolymer system composed of the side-chain LC polymer and amorphous polymer, the properties of two polymer classes will be closely bound to each other and then the following combined effects can be considered. ${ }^{10-20}$

1. The connection of two polymer chains at the interface may affect the orientational and positional orders of LC phase.

2. The global chain conformation of LC segment which changes on the crystal-LC and LC-isotropic phase transitions may affect the size and type of the microphase separation.

3. The size and type of microphase separation may affect the phase transition of LC segment.

In this paper, we prepared the block copolymers composed of the polystyrene and LC poly(1) segments with various molecular weights by sequential living polymerization and examined the structural aspects raised above.

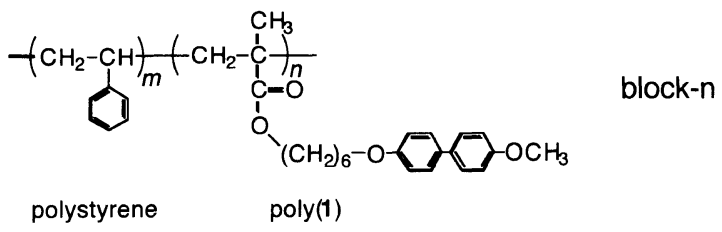

EXPERIMENTAL

\section{Materials}

The A-B block copolymers were prepared by a sequential polymerization of styrene as monomer $A$ and 6-[4-(4-methoxyphenyl)phenoxy]-hexyl methacrylate 
Table I. Block copolymerization of styrene with (MPPHM) ${ }^{\mathrm{a}}$

\begin{tabular}{lrcc}
\hline & \multicolumn{2}{c}{ Polystyrene-Block-poly(1) } & \\
\cline { 2 - 3 } Sample & \multicolumn{1}{c}{$M_{w} / M_{n}{ }^{\mathrm{c}}$} \\
& $M_{n}$ (Calcd) & $M_{n}(\text { Obsd })^{\mathrm{b}}$ & \\
\hline Block-1 & $3800-3600$ & $3800-3700$ & 1.07 \\
Block-2 & $4800-4400$ & $4600-4800$ & 1.04 \\
Block-3 & $5800-5200$ & $5800-5200$ & 1.12 \\
Block-4 & $8000-9000$ & $8000-11400$ & 1.05 \\
Block-5 & $9400-9500$ & $9300-10700$ & 1.05 \\
Block-6 & $12000-13000$ & $12000-15500$ & 1.04 \\
Block-7 & $13000-13000$ & $13300-15500$ & 1.04 \\
Block-8 & $14000-12000$ & $15000-15000$ & 1.04 \\
Block-9 & $18000-14000$ & $18700-15000$ & 1.03
\end{tabular}

${ }^{\text {a }}$ Block copolymerization was carried out by sequential addition of styrene at first and then (1) in THF at $-78^{\circ} \mathrm{C}$ with $s$-BuLi as an initiator. Yields of polymers were quantitative in all runs. Polymerization times at the first and at the second stage were 0.2 and $4 \mathrm{~h}$, respectively. ${ }^{\mathrm{b}} M_{n}$ (obsd) of poly(1) segment was determined by ${ }^{1} \mathrm{H}$ NMR. ${ }^{\mathrm{c}} M_{w} / M_{n}$ was determined from SEC profile based on the calibration of standard polystyrene.

(MPPHM) as monomer B. Living polystyrene was first prepared with $s$-BuLi in tetrahydrofuran (THF) at $-78^{\circ} \mathrm{C}$ for $10 \mathrm{~min}$ and then 1,1-diphenylethylene was added to cap the highly reactive polystyryl anion. The sequential polymerization of MPPHM was then carried out with the polymeric anion in the presence of $\mathrm{LiCl}$ in THF at $-40^{\circ} \mathrm{C}$ for $2 \mathrm{~h}$. The polymerization was usually homogeneous but in some cases became heterogeneous because of the polymer precipitation. This is dependent on the molecular weight of the LC poly(1) block; the increase of the molecular weight of poly(1) tends to make the system heterogeneous. Thus, the molecular weight of the poly(1) segment in this study was limited upto 20000 and nine block copolymers with various molecular weights from 8000 to 35000 and with the composition of segments around $50 \mathrm{wt} \%$ were prepared. The copolymers were designated here "block- $n$ " $(n=1-9$; the sample number). The composition of each segment was finally determined by ${ }^{1} \mathrm{H}$ NMR, and the $M_{n}$ and $M_{w} / M_{n}$ values were estimated from a SEC profile based on the standard polystyrene calibration. As summarized in Table I, the polymers in all cases were obtained with quantitative yields, possessing the predictable $M_{n}$ values, compositions, and narrow molecular weight distributions. ${ }^{1} \mathrm{H}$ NMR showed that the syndiotacticity of all the poly(1) blocks obtained here is around $80 \%$.

\section{Methods}

Differential scanning calorimetric (DSC) measurements were carried out with a Perkin-Elmer DSC Model II at a scanning rate of $2^{\circ} \mathrm{C} \mathrm{min}-1$.

$\mathrm{X}$-Ray measurements were performed by using a Rigaku Denki X-ray generator with $\mathrm{Ni}$-filtered $\mathrm{Cu}-\mathrm{K}_{\alpha}$ radiation. Temperatures of the sample were regulated within $1^{\circ} \mathrm{C}$ by using a Mettler FP- 82 hot stage. Reflection spacings were calibrated by using a silicon standard.

The TEM observation to clarify the morphology of block copolymers was performed by a Hitachi H-500 transmission electron microscope with $100 \mathrm{kV}$ of accelerating voltage. For this observation, an ultrathin section of the polymer film was prepared as follows. The polymer film was dipped in a $1 \mathrm{wt} \%$ aqueous solution

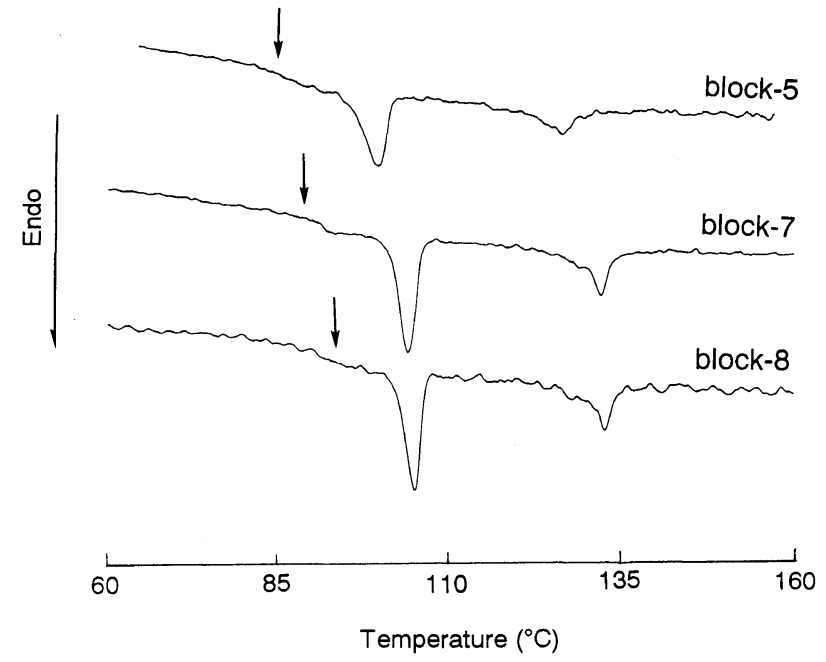

Figure 1. DSC thermograms of block copolymers measured at a heating rate of $2{ }^{\circ} \mathrm{Cmin}^{-1}$. Two peaks observed are attributed to the crystal- $S_{\mathrm{A}}$ and $S_{\mathrm{A}}$-isotropic phase transitions of poly(1) segment. The arrow indicates the glass transition of polystyrene segment.

Table II. DSC data of polystyrene-block-poly(MPPHM) ${ }^{a}$

\begin{tabular}{|c|c|c|c|c|}
\hline \multirow{3}{*}{ Sample } & \multicolumn{4}{|c|}{$\begin{array}{c}\text { Transition temperature } /{ }^{\circ} \mathrm{C} \\
\text { corresponding enthalpy changes } / \mathrm{kcal} \mathrm{mol}^{-1 \mathrm{~b}}\end{array}$} \\
\hline & \multicolumn{2}{|c|}{ Heating } & \multicolumn{2}{|c|}{ Cooling } \\
\hline & $T_{1}\left(\Delta H_{1}\right)$ & $T_{2}\left(\Delta H_{2}\right)$ & $T_{2}\left(\Delta H_{2}\right)$ & $T_{1}\left(\Delta H_{1}\right)$ \\
\hline Block-1 & $97.1(0.62)$ & $106.3(0.52)$ & $100.7(0.41)$ & $90.9(0.59)$ \\
\hline Block-2 & $84.4(0.26)$ & $111.2(0.19)$ & $109.9(0.11)$ & $88.1(0.24)$ \\
\hline Block-3 & $88.6(0.30)$ & $112.2(0.28)$ & $111.7(0.30)$ & $85.4(0.28)$ \\
\hline Block-4 & $102.3(0.68)$ & $129.8(0.34)$ & $129.1(0.42)$ & $98.4(0.68)$ \\
\hline Block-5 & $100.1(0.72)$ & $128.5(0.38)$ & $127.8(0.26)$ & $96.9(0.68)$ \\
\hline Block-6 & $105.4(0.79)$ & $133.4(0.32)$ & $132.9(0.47)$ & $100.7(0.79)$ \\
\hline Block-7 & $103.9(0.80)$ & $131.9(0.40)$ & $131.3(0.42)$ & $99.6(0.76)$ \\
\hline Block-8 & $105.4(0.76)$ & $132.8(0.44)$ & $132.1(0.44)$ & $101.0(0.76)$ \\
\hline Block-9 & $104.3(0.77)$ & $132.5(0.45)$ & $132.0(0.45)$ & $100.4(0.77)$ \\
\hline
\end{tabular}

${ }^{a}$ Determined by DSC measurement at 2nd heating and cooling $\left(2^{\circ} \mathrm{Cmin}^{-1}\right) . \quad{ }^{\mathrm{b}}$ Estimated per mole of poly(1) segment.

of ruthenium tetraoxide $\left(\mathrm{RuO}_{4}\right)$ as a staining reagent for $20 \mathrm{~min}$. After being dried, the film was embedded in an epoxy resin and cut into ultrathin sections $(700-1000 \AA$ thick) by an ultramicrotome with diamond knife. The sectioned specimens were further stained with the vapor of $\mathrm{RuO}_{4}$ for $5 \mathrm{~min}$ before observation.

\section{RESULTS AND DISCUSSION}

\section{Transition Behavior of Block Copolymers}

DSC thermograms of the copolymers are shown in Figure 1. All the copolymers exhibit the two transitions, similarly as the homopolymers of poly(1). ${ }^{9}$ The phase sequence is also similar; the crystal- $S_{\mathrm{A}}$ and $S_{\mathrm{A}}$-isotropic phase transitions take place. The well-defined thermotropic behavior due to the poly(1) segment shows that there is formed a segregation structure into amorphous polystyrene and LC poly(1) microphases. The transition temperatures and enthalpies are summarized in Table II.

On comparison with the homopolymers, the block copolymers show several distinct features with respect to the transition behavior. In Figure $2 a$, the transition temperatures are plotted against the molecular weight 


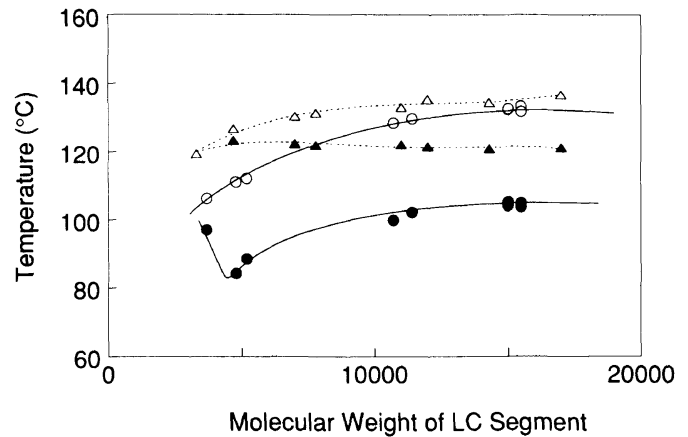

(a)

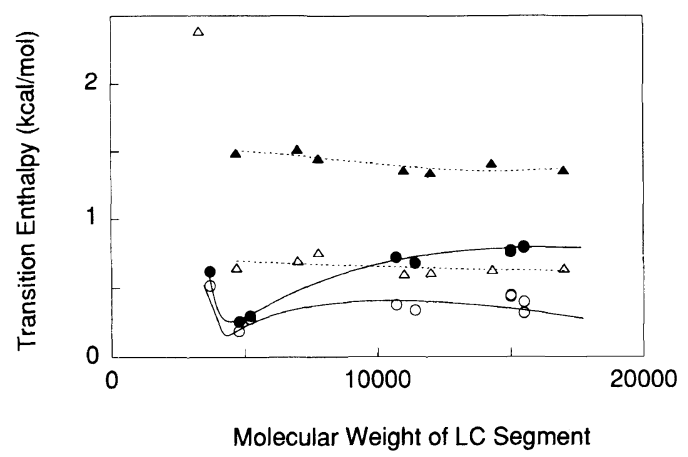

(b)

Figure 2. Dependence of (a) the transition temperatures and (b) transition enthalpies on the degree of polymerization (DP) of poly(1) block. The data for the block copolymers are given by the circles and are compared with the data of the homopolymers (the triangles). The open symbols correspond to the $S_{\mathrm{A}}$-isotropic transition while the closed ones correspond to the crystal- $S_{\mathrm{A}}$ transition.

LC poly(1) segment and compared with those of homopolymers having a corresponding molecular weight. From this figure, one can find at once that the smectic temperature region is fairly expanded in the block copolymers. This expansion results mainly from a depression in the crystal melting temperature since there can be seen no significant difference in the isotropization temperature of the $S_{\mathrm{A}}$ phase.

We can also find in Figure $2 b$ that the transition enthalpies $(\Delta H)$ of both transitions are significantly smaller than those of homopolymers. ${ }^{9}$ The enthalpy change of crystal melting is roughly half of that in the homopolymer. Since the microphase domain boundary is sharp (Figure 3), this may be caused by an irregular structure at the interface; the poly(1) segment placed near to the phase boundary is in a disordered state and only the segment in the interior of its microphase takes part in the crystallization. This effect is somewhat weakened in the $S_{\mathrm{A}}$ phase since the value of $\Delta H$ for its isotropization is smaller by approximately $40 \%$ than that of the homopolymer ${ }^{9}$ (Figure 2b).

\section{Microdomain Morphology of Block Copolymers}

Figure 3 represents a typical electron micrograph for ultrathin section cut out from the film of block-6. It clearly demonstrates the microphase separation, with the LC poly(1) microphases appearing dark because of staining with $\mathrm{RuO}_{4}$. The phase boundary is very sharp, and the basic morphology is a lamellar type. The lamellar

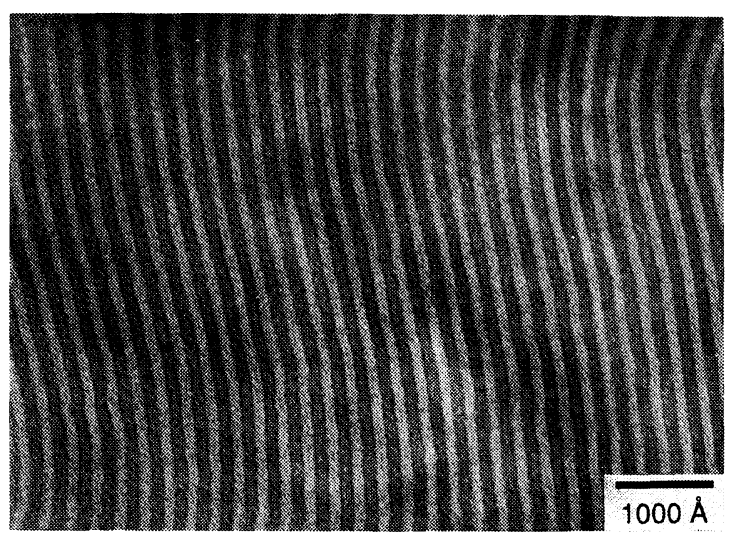

Figure 3. Typical TEM photograph which was observed for ultrathin section cut out from the cast film of block-6 and stained with $\mathrm{RuO}_{4}$.

Table III. Lamellar spacings $(\AA)$ of the block copolymers

\begin{tabular}{|c|c|c|c|c|c|c|}
\hline \multirow{2}{*}{ Sample } & \multicolumn{5}{|c|}{ Crystalline phase } & \multirow{2}{*}{$\begin{array}{c}\text { Isotropic Phase } \\
D_{\mathrm{x}} \\
(\mathrm{SAXS})\end{array}$} \\
\hline & $\begin{array}{c}D_{\mathrm{m}} \\
(\mathrm{TEM})\end{array}$ & $\begin{array}{c}D_{\mathrm{x}} \\
\mathrm{SAXS})\end{array}$ & $d_{\mathrm{a}}$ & $d_{\mathrm{lc}}$ & $L_{\mathrm{lc}}^{\mathrm{a}}$ & \\
\hline Block-1 & 75 & 101 & 52 & 49 & 25 & \\
\hline Block-2 & 115 & 130 & 70 & 60 & 33 & \\
\hline Block-3 & 110 & 137 & 78 & 59 & 35 & \\
\hline Block-4 & 190 & 191 & 91 & 100 & 78 & 145 \\
\hline Block-5 & 170 & 198 & 119 & 79 & 73 & 154 \\
\hline Block-6 & 200 & 235 & 118 & 117 & 105 & 185 \\
\hline Block-7 & 200 & 230 & 120 & 110 & 105 & 180 \\
\hline Block-8 & 250 & 252 & 141 & 111 & 103 & 192 \\
\hline Block-9 & 250 & 258 & 159 & 99 & 103 & 202 \\
\hline
\end{tabular}

${ }^{a}$ Extended chain length of poly(1) segment (see the text).

type of morphology is observed for all other specimens as expected from the weight fractions of the two blocks around $50 \% .^{21}$ The lamellar spacings by this method, $D_{\mathrm{m}}$, are listed in Table III.

The lamellar type of microphase separation was also observed from a small-angle X-ray pattern which includes a sharp reflection. The reflection spacings $\left(D_{x}\right)$ are collected in Table III. One can find a quantitative correspondence between the lamellar spacings of $D_{\mathrm{m}}$ and $D_{\mathrm{x}}$ due to both methods.

The lamellar size of each styrene or poly(1) segment, $d_{\mathrm{a}}$ or $d_{\mathrm{lc}}$, can be determined from the microphotographs, but the evaluation by this method has some ambiguity since the lamellar size observed in the microtomed film depends on the cut angle to the lamellar normal. The values of $d_{\mathrm{a}}$ and $d_{\mathrm{lc}}$ listed in Table III were thus obtained by using the relative ratio of $d_{\mathrm{a}}$ and $d_{\mathrm{lc}}$ in the TEM observation and then regarding $D_{\mathrm{x}}$ as a whole lamellar size. These values of $d_{\mathrm{a}}$ and $d_{\mathrm{lc}}$ correspond to those expected from the compositions of segments under the assumption of an incompressibility of medium.

\section{Microstructure in Crystal and Smectic A Phases of Poly(1) Block}

Figures $4 \mathrm{a}, 4 \mathrm{~b}$, and $4 \mathrm{c}$ show the wide-angle X-ray patterns taken for the crystal, $S_{\mathrm{A}}$ and isotropic phases of poly(1) block, respectively. The oriented fiber specimen used here was prepared by pulling up the isotropic melt and its axis is placed in the vertical direction. The re- 


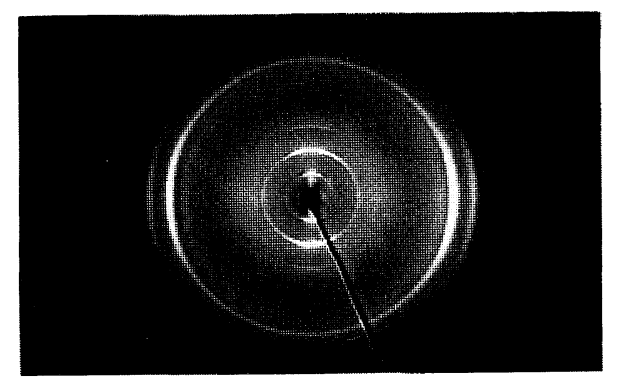

(a)

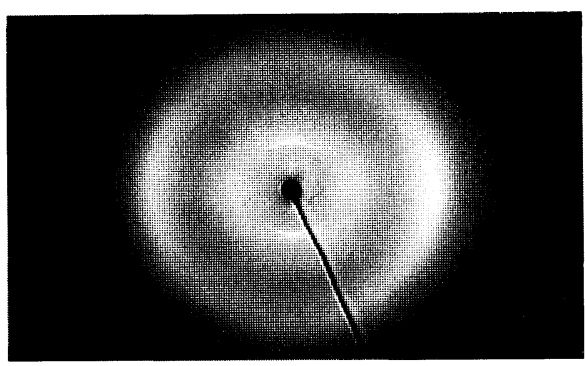

(b)

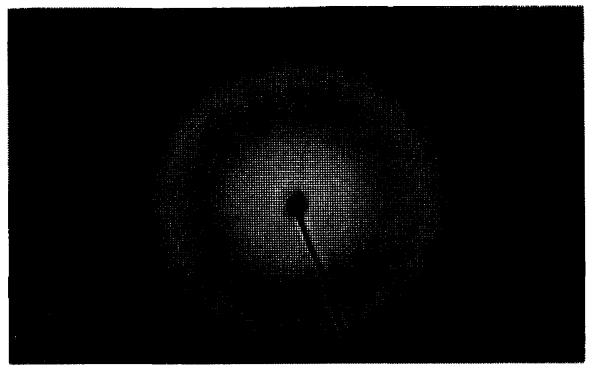

(c)

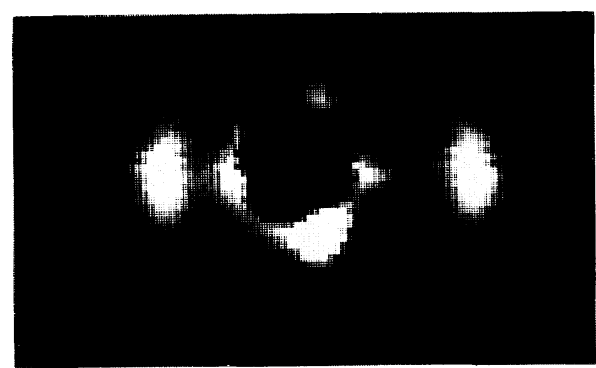

(d)

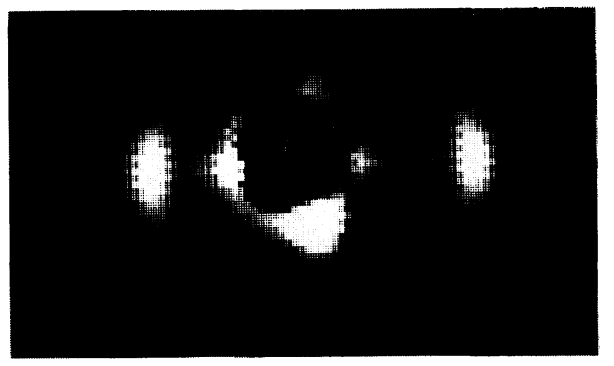

(e)

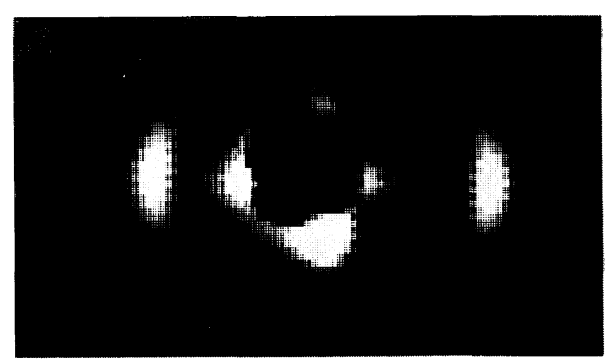

(f)

Figure 4. Wide-angle X-ray patterns for the crystal, $S_{\mathrm{A}}$ and isotropic phases of poly(1) block are given in (a), (b), and (c), respectively. (d), (e), and (f) are the corresponding small-angle X-ray patterns. Here, the fiber specimen was prepared by drawing the isotropic melt of block-7, and its axis is placed in the vertical direction. The oriented patterns observed here are invariably observed on heating and coolig cycles.

Table IV. X-Ray spacing $(\AA)$ of the crystalline and smectic A phases in block-8

\begin{tabular}{cc}
\hline Crystal & Smectic A \\
\hline \multicolumn{3}{c}{ Small-angle region } \\
$25.8(001)^{\mathrm{a}} \mathrm{m}$ & $26.4(001) \mathrm{vvw}$ \\
$12.8(002) \mathrm{vs}$ & $13.2(002) \mathrm{m}$ \\
$8.58(003) \mathrm{w}$ & \\
& \\
$4.48(110) \mathrm{s}$ & Wide-angle region \\
$3.99(200) \mathrm{m}$ & \\
$3.22(210) \mathrm{w}$ & \\
$2.58(120) \mathrm{vw}$ & \\
$2.38(310) \mathrm{vw}$ &
\end{tabular}

${ }^{a}$ Indices are based on the lattice noted in the text.

flection spacings observed for crystal and $S_{\mathrm{A}}$ phases are listed in Table IV.

The crystalline pattern of Figure $4 \mathrm{a}$ includes three inner reflections along a meridian and also several reflections along an equator. These reflections are commonly observed for all copolymers and can be indexed by the orthorhombic unit cell with parameters of $a=$ $8.00 \AA, b=5.41 \AA$, and $c=25.8 \AA^{9}$ (refer to Table IV), in which two repeat units are included.
Although the three-dimensional unit cell is given here for convenience, the structure is of the layer type rather than of three-dimensional order since only $(h k 0)$ and $(00 l)$ reflections are observed. Within an individual layer, the side-chain biphenyl groups are crystallized into the two-dimensional lattice with $a=8.00 \AA, b=5.41 \AA$, and $\gamma=90^{\circ}$ like in the biphenyl crystal. ${ }^{22}$ These layers are piled up with a spacing of $c=25.8 \AA$.

As reported previously, ${ }^{9}$ the two following facts,

1. The layer spacing of $25.8 \AA$ corresponds roughly to the length of the side chain in an extended form.

2. The (002) layer reflection is much more intense than the (001) one (Figure $4 a$ ), showing that there are two maxima of electron density in a repeating length of $25.8 \AA$ along the layer normal.

lead to a plausible layered structure in which there is a segregation into two regions of side-chain mesogens and main-chain backbones. In other words, the main chains are confined in the interplane between the mesogenic side-chain layers and so on average half of side chains in each layer are connected to one of the main chains which lie in this plane. The tentative layer structure is illustrated in Figure 5 where the open and closed ellipsoids show upward- and downward-directed meso- 


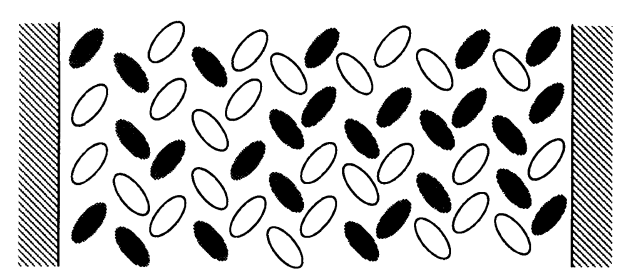

(a)

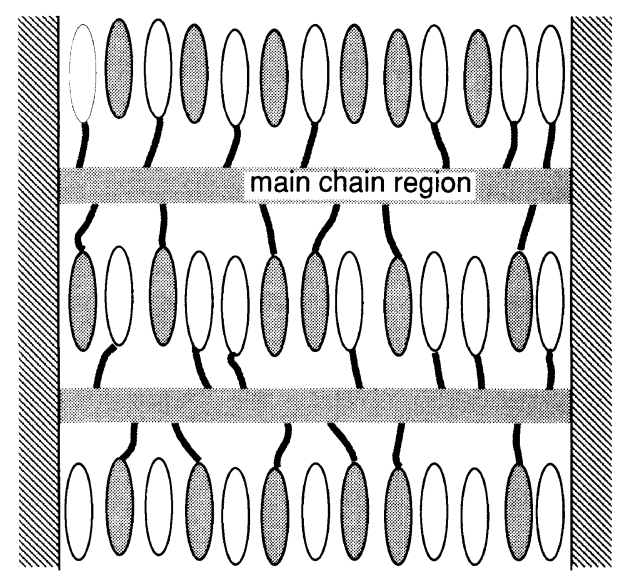

(b)

Figure 5. Schematic illustration of the packing structure of side-chain mesogenic groups in the crystalline phase which can be elucidated from the X-ray patterns of Figure 4. Parts (a) and (b) are viewed parallel and perpendicular to the long axis of the mesogenic groups, respectively. The open and closed ellipsoids show the mesogenic groups directed upward and downward, respectively.

gens, respectively. Such a type of layered structure is often observed in this class of side-chain LC polymers. ${ }^{23-25}$

On heating the sample to the $S_{\mathrm{A}}$ temperatures, the outer reflection on the equator becomes broad with a spacing of $4.5 \AA$ (Figure $4 \mathrm{~b}$ ), while the meridional layer reflections are still sharp. The spacing of the first layer reflection is $26.4 \AA$ so that the layer thickness of $S_{\mathrm{A}}$ phase is approximate to that in the crystalline phase. Figure 6a shows the intensity profiles of the layer reflections measured at various temperatures and Figure $6 \mathrm{~b}$ shows the temperature dependence of the intensity of the (002) reflection. While the intensity is not much varied with the temperature in the crystalline phase, it decreases remarkably with the temperature in the $S_{\mathrm{A}}$ phase. This means that the layer order of $S_{\mathrm{A}}$ phase decreases remarkably with the increasing temperature. ${ }^{26}$

On further heating to isotropic melt, all of the sharp reflections disappear and the resulting broad reflections indicate no orientation of molecules as expected (Figure $4 c)$.

\section{Temperature Dependence of Microdomain Morphology}

Figures $4 \mathrm{~d}, 4 \mathrm{e}$, and $4 \mathrm{f}$ show the small-angle X-ray patterns taken for the same sample of Figures $4 a, 4 b$, and $4 \mathrm{c}$, respectively. There can be seen the lamellar reflection on the equator, showing the preferential orientation with the microphase lamellae lying along the fiber axis. Further, it can be concluded from a comparison of the wide-angle and small-angle X-ray patterns

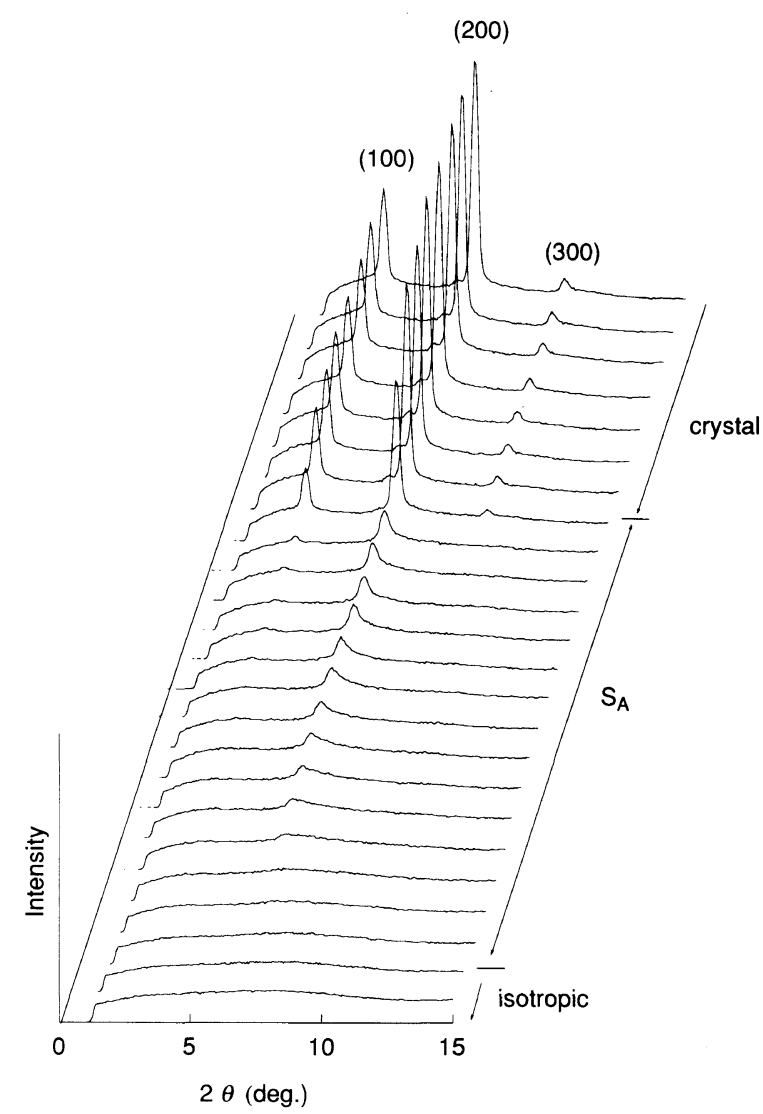

(a)

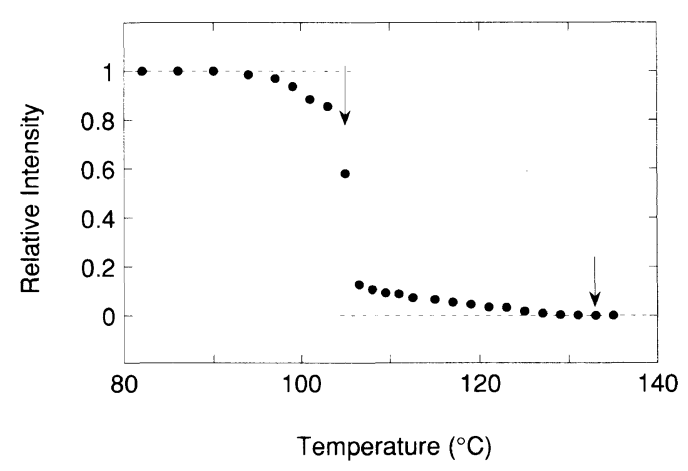

(b)

Figure 6. Temperature dependence of (a) the intensity profiles of the layer reflections with indices of (001), (002), and (003), and (b) the relative intensity of $(002)$ reflection.

that the crystal and smectic layers of LC poly(1) segments are oriented normal to the supermolecular lamellae as schematically illustrated in Figure 5. At this time, we suppose that this correlation is promoted by a tendency of the main-chain backbone to orient perpendicular to the interface. ${ }^{9,10}$

Figure 7 shows the temperature dependence of the lamellar spacing, $D_{\mathrm{x}}$. The temperatures of the crystal- $S_{\mathrm{A}}$ and $S_{\mathrm{A}}$-isotropic phase transitions are given by dashed lines in the same figures. If the molecular weights are relatively lower than 10000 (Figure 7, (a)), the lamellar type of microphase separation disappears just as the transition of $S_{\mathrm{A}}$ to isotropic phase takes place. This compatibility may be achieved by an appreciable gain of combinatorial entropy due to the isotropization of LC poly(1) segment. For the higher molecular weight 


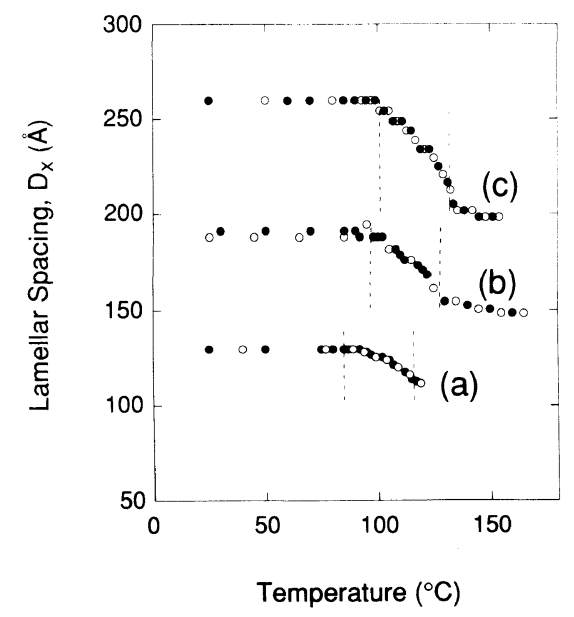

Figure 7. Temperature dependence of the microphase lamellar spacing, $D_{\mathrm{x}}$, observed for (a) block-2, (b) block-5, and (c) block-8. The open and closed circles were measured on heating and cooling, respectively.

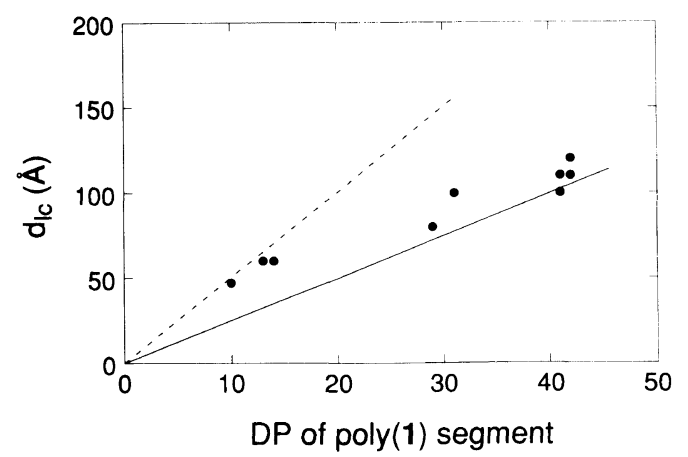

Figure 8. Dependence of the lamellar thickness, $d_{1 \mathrm{c}}$, on the degree of polymerization (DP) of poly(1) segment. The solid and dashed lines correspond to $L_{\mathrm{lc}}$, the extended chain length of poly(1) segment, and $2 L_{\mathrm{lc}}$, respectively.

samples, on the other hand, the lamellar type of microphase is invariably observed through the whole temperature region from crystal to isotropic melt.

The most distinct feature observed here is that the lamellar spacing decreases remarkably through the $S_{\mathrm{A}}$ temperature region, although it is relatively constant in the crystal and isotropic phases (Figure 7, (b) and (c)). Overall change is as large as one-fifth of the original lamellar spacing and completely reversible on heating and cooling cycles, indicating that it proceeds at a thermodynamic equilibrium. Considering that the uniaxial orientation of side-chain mesogens is not essentially altered on the transition from crystal to $S_{\mathrm{A}}$ and also through the $S_{\mathrm{A}}$ temperature region, such a distinct variation in the lamellar spacing as well as in the smectic layer order is obviously caused by the conformational change in the main-chain backbone of LC poly(1) segment.

\section{Main-Chain Conformation of Poly(1) Segment}

In the side-chain LC polymers, the global conformation of the backbone has become a central issue. In a $S_{\mathrm{A}}$ phase, we can easily visualize that the backbone adopts an oblate conformation. This can be understood by considering the confinement effect exerted by a smectic field on the backbones; since the existence of $S_{\mathrm{A}}$ phase usually proceeds from a tendency of the aromatic and

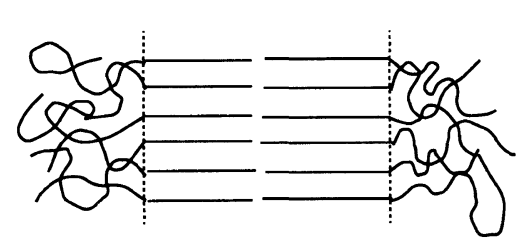

(a)

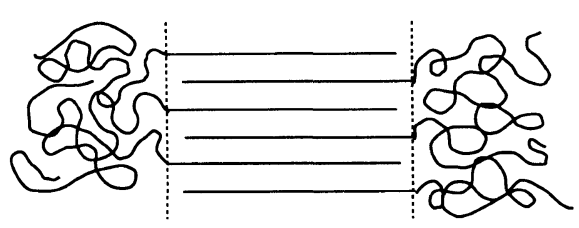

(b)

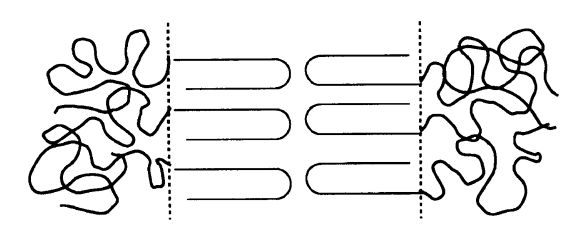

(c)

Figure 9. Illustration of the main-chain structures of poly(1) segment in the microphase. In model (a) which can be expected for the low molecular weight specimens, the main chains sticking out of the neighboring interfaces are fully extended and fill the space without intercalating to each other. In models (b) and (c) which can be expected for the higher molecular weight, the main chains in an extended form are intercalating to each other or folded back.

aliphatic moieties to segregate, then the backbone should be preferentially located in the aliphatic part of the smectic layers. Further, the segregation tendency may be favorable since it is likely that the crossing of the main chain from layer to layer produces a significant deffect. Small-angle neutron scattering on mixtures of unlabelled and labelled polymers has proved to be a very efficient method to study this point experimentally. ${ }^{27-34}$ This technique has clearly established in several cases that the backbone adopts an oblate conformation in smectic A phase; the backbone might be considered as an anisotropic coil with $R_{/ /}<R_{\perp}$, where $R_{/ /}$and $R_{\perp}$ are the radii of gyration of the backbone parallel and perpendicular to the director of the side-chain mesogens, respectively. ${ }^{27-33}$ In the present system where one end of LC segment is confined at the interface, the main-chain conformation can be examined through the change in the size of lamellar microphase domain.

At first we refer to the main-chain conformation in the crystalline phase. This can be examined by comparing the lamellar thickness of $d_{1 \mathrm{c}}$ with the extended chain length of the poly(1) segment $\left(L_{\mathrm{lc}}\right)$. By using $2.5 \AA$ as a repeat length of poly(1), the value of $L_{\mathrm{lc}}$ can be obtained as listed in Table III. In Figure 8, the values of $d_{\mathrm{lc}}$ are plotted against the degree of polymerization (DP) of poly(1) and compared with the values of $L_{\mathrm{lc}}$. The interesting result obtained is that $d_{\mathrm{lc}}$ is as large as twice of $L_{\mathrm{lc}}$ in the low molecular weight copolymers, block-1, block-2, and block-3. This means that the main chains sticking out of the neighboring interfaces should be fully extended and fill the space without intercalating to each other as illustrated in Figure 9a. The further argument 


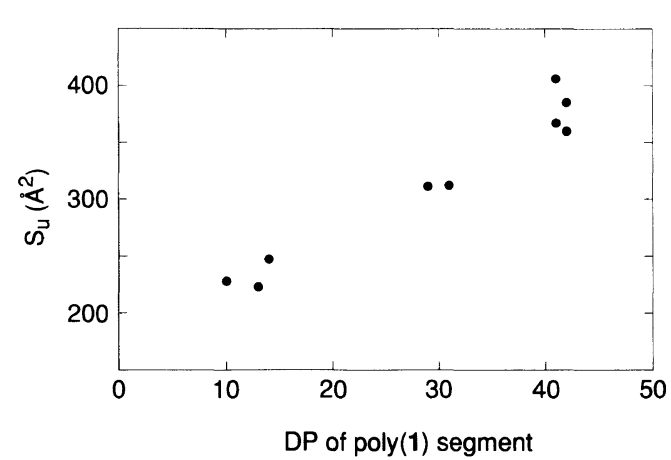

Figure 10. Dependence of the interfacial area occupied by a block polymer chain, $S_{u}$, on the degree of polymerization (DP) of poly(1) segment.

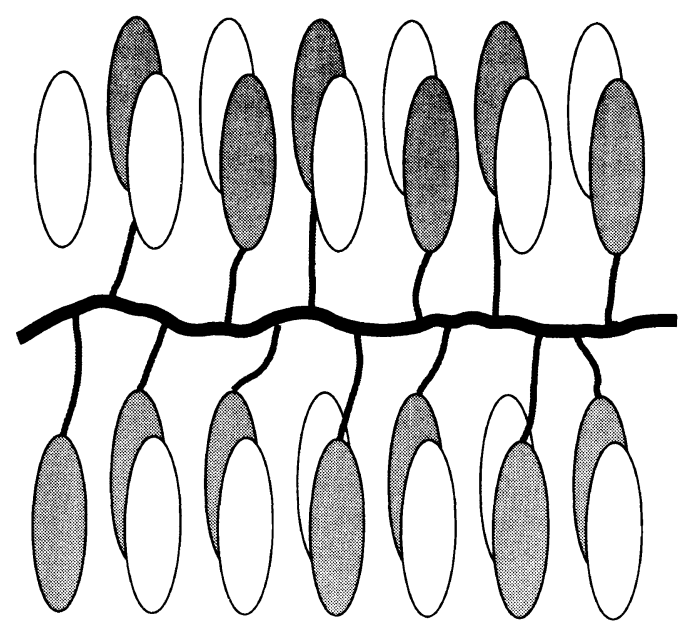

Figure 11. Schematic illustration of the crystalline structure in which the extended main chain is confined in the space between the side-chain mesogenic layers. Half of the side chains participate to form the upper layer and another half take part in the lower layer. The distance between the adjacent mesogenic groups included in each smectic layer and sticking out of one chain is of the order of $5 \AA$.

on the main-chain conformation can be done on the basis of the interfacial area occupied by a block polymer chain $\left(S_{\mathrm{u}}\right)$. Figure 10 shows the values of $S_{\mathrm{u}}$ estimated by using the density of poly $(\mathbf{1}),{ }^{9} 1.17 \mathrm{~g} \mathrm{ml}^{-1}$, as a function of the DP of poly(1). In the low molecular weight block-1, block-2 and block-3, the values of $S_{\mathrm{u}}$ are ranged from 200 to $250 \AA^{2}$. Since the crystal layer lies perpendicular to the interface and its layer spacing is $25.4 \AA$, the interfacial area should be orthogonal with one edge of $25 \AA$ and another edge of $8-10 \AA$. Coupling these values with the crystal lattice of the mesogenic groups and considering that half of the side chains in each layer have to be connected to one of main chains (refer to Figure 5), we reach the conclusion that the distance between adjacent mesogenic groups included in each layer and sticking out of one chain should be of the order of $5 \AA$. This value can be explainable only when the main chain is in an extended form as illustrated in Figure 11. Although the relative orientation of side chains and main chains depends on the length of the flexible spacer, such a highly oriented conformation of main chain has been postulated from the ${ }^{2} \mathrm{H}$ NMR study by Boeffel et al. ${ }^{35}$

As the molecular weight increases, the values of $d_{\mathrm{lc}}$ are approximate to $L_{\mathrm{lc}}$ (Figure 8 ) and the values of $S_{\mathrm{u}}$ becomes as large as $400 \AA^{2}$, almost twice of those of low
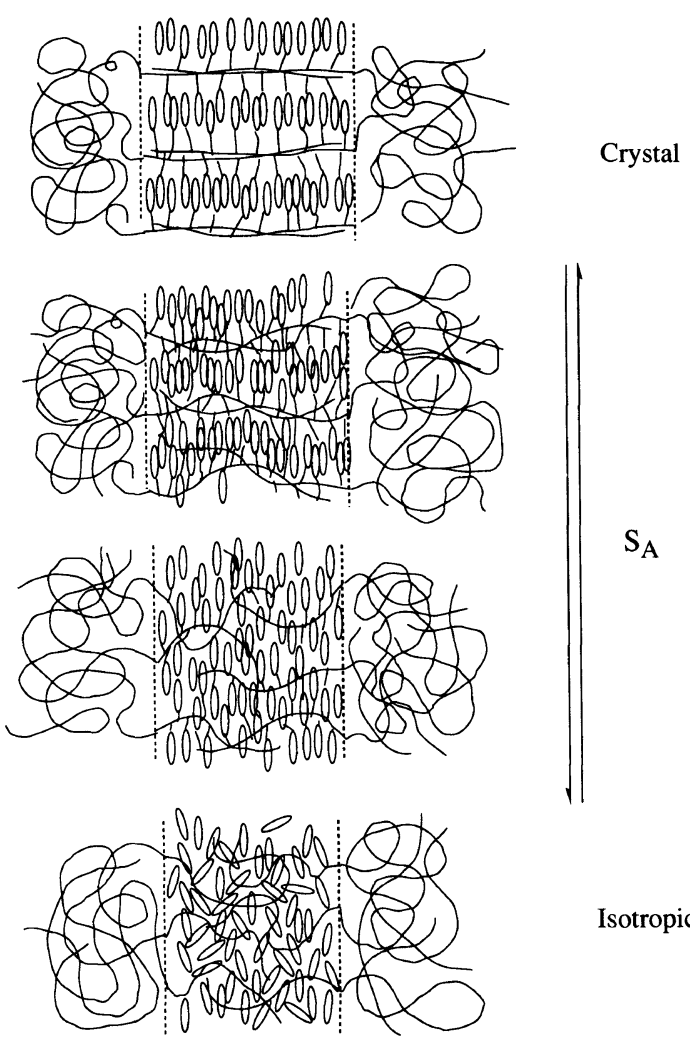

Isotropic

Figure 12. Schematic illustration for the change of the overall structure which takes place on increasing temperature.

molecular weight polymers (Figure 10). These can be expected if the chains in the extended form are intercalating to each other or folded back as illustrated in Figures $9 b$ or 9c, respectively. Such a situation has been encountered for the crystallizable block copolymers. ${ }^{36-38}$

Next we consider the temperature dependence of the lamellar size. As previously observed in Figure 7, the lamellar domain size in the $S_{\mathrm{A}}$ phase changed continuously from the size of the crystalline phase to that of the isotropic phase. All the specimens exhibited the $20-25 \%$ reduction of the lamellar thickness as found in Table III. Since it is obvious that the main chains of poly(1) segment take up the random coil in the isotropic phase, this variation can be obviously attributed to the conformational change of the main-chain from an extended form to random coil as illustrated schematically in Figure 12. The obvious conformation for a main chain to have in order to minimize the packing energy of mesogenic side-chain groups into a $S_{\mathrm{A}}$ layer is to lie entirely in a plane between layers like in the crystal. Such a conformation can be expected at the lower temperatures far apart from the isotropization temperature of $S_{\mathrm{A}}$. On the other hand, the entropic force, leading to coiled main chain which has to cross through one or several layers on the average, will be important at the elevated temperatures. Such a crossing is energetically unfavorable for the side chains since along the crossing of the main chain the side chains cannot be arranged in such a way that they fit into the smectic layers. Note that the distance between two adjacent side chains is of the order of $5 \AA$ whereas the thickness of one layer is around $25 \AA$. Therefore, at least three or four side chains cannot match the smectic layer order during each crossing, resulting in 
the defect. The significant decrease in the layer order of $S_{\text {A }}$ phase with the increasing temperature as observed in Figure 6 may be due to this effect. Thus, the distinct temperature variation of $D_{\mathrm{x}}$ can be underestood as resulting from the interplay between the tendency for the side chains to form the ordered smectic layer and the tendency for the main chains to assume the random coil conformation. Considering the exponential temperature dependence of $D_{\mathrm{x}}$ and according to the theory, ${ }^{39-42}$ we have the equation

$$
D_{\mathrm{x}} \propto \exp \left(-E / k_{\mathrm{B}} T\right)
$$

where $E$ is regarded as the energy making each crossing or the energy making a smectic deffect. In the present system, we find the energy of around $2.5 \mathrm{kcal} \mathrm{mol}^{-1}$. This energy is of the same order as those reported. ${ }^{30}$

\section{CONCLUDING REMARKS}

We have synthesized the A-B block copolymers with polystyrene as A segment and side-chain LC polymer as B segment by sequential living polymerization. The polymerization successfully proceeded with symmetrical unimodal distribution and the resulting copolymers possessed the predictable $M_{n}$ values, compositions, and narrow molecular weight distributions.

All the copolymers employed, having the compositions of segments around $50 \mathrm{w} \%$ and the various molecular weights of 8000 to 35000 , exhibit the lamellar type of segregation. The side-chain LC polymer in the microdomain forms crystal, $S_{\mathrm{A}}$ and isotropic phases. This thermotropic phase behavior is essentially the same as that of the homopolymer although the smectic temperature region expanded mainly due to the depression of crystal melting. The lamellar type of microphase segregation is maintained over the whole temperature region from the crystal to isotropic phase if the samples have the higher molecular weights. The layered structures of crystal and $S_{\mathrm{A}}$ phases are formed with a preferential orientation of the side-chain mesogens lying parallel to the microdomain interface.

The lamellar thickness is dependent on the $S_{\mathrm{A}}$ temperature. With increasing temperature, it decreases from the lamellar thickness of the crystal phase to that of the isotropic phase and no jump can be seen on the crystal$S_{\mathrm{A}}$ and $S_{\mathrm{A}}$-isotropic transitions. The reduction is about $20-25 \%$, which can be caused by the conformational change in the main chain of LC segment from the extended form to the random coil. The space filling model dictates that to produce the orientational and positional orders of the side-chain mesogens in the $S_{\mathrm{A}}$ phase the main chains should assume an extended conformation and be placed in the plane between the side chain layers. On the other hand, the random coil of main chains would be entropically favored, but it can produce significant defects in a smectic structure since the coiled main chain has to cross one or more smectic layers. Thus, the temperature dependence of main-chain conformation is considered to arise as a result of the counterbalance between its energetical cost and entropy gain. Considering the exponential temperature dependence of lamellar thickness, we estimate the activation energy of around $2.5 \mathrm{kcal} \mathrm{mol}^{-1}$, which is regarded as the energy making a smectic deffect.

\section{REFERENCES}

1. C. B. McArdle, "Side Chain Liquid Crystal Polymers," Chapman and Hall, New York, N.Y., 1989.

2. A. A. Collyer, "Liquid Crystal Polymers," Elsevier Applied Science, London and New York, 1992.

3. V. Percec, D. Tomazos, and C. Pugh, Macromolecules, 22, 3259 (1989).

4. V. Percec and M. Lee, Macromolecules, 24, 1017 (1991); ibid., 24, $2780(1991)$.

5. Z. Sagane and R. M. Lenz, Macromolecules, 22, 3763 (1989).

6. Z. Komiya, C. Pogh, and R. R. Schrock, Macromolecules, $\mathbf{2 5}$, 3609 (1992); ibid., 25, 6586 (1992).

7. E. Papon, A. Deffieux, F. Hardouin, and M. F. Achard, Liq. Cryst., 11, 803 (1992).

8. R. Bohnert, H. Fikelmann, and P. Lutz, Makromol. Chem., Rapid Commun., 14, 139 (1993).

9. M. Yamada, T. Iguchi, A. Hirao, S. Nakahama, and J. Watanabe, Macromolecules, 28, 50 (1995).

10. J. Adams and W. Gronski, Makromol. Chem., Rapid Commun., 10, 553 (1989).

11. M. Hefft and J. Springer, Makromol. Chem., Rapid Commun., 11, 397 (1990).

12. B. Zaschke, W. Frank, H. Fischer, K. Schmultzler, and M. Arnold, Polym. Bull., 27, 1 (1991).

13. V. Percec, and M. Lee, J. Macromol. Sci. Chem., 29, 723 (1992).

14. Z. Komiya and R. R. Schrock, Macromolecules, 26, 1387 (1993).

15. R. Bohnert and H. Finkelmann, Makromol. Chem. Phys., 195, 689 (1994)

16. M. Arnold, S. Poser, H. Fischer,W. Frank, and H. Utschick, Macromol. Chem., Rapid Commun., 15, 487 (1994).

17. H. Fischer, S. Poser, M. Arnold, and W. Frank, Macromolecules, 27, 7133 (1994).

18. H. Fischer, Polymer, 35, 3786 (1994).

19. J. Adams, J. Sanger, C. Tefehne, and W. Gronski, Macromol. Chem., Rapid Commun., 15, 879 (1994).

20. H. Fischer, S. Poser, and M. Arnold, Liq. Cryst., 18, 503 (1995).

21. F. S. Bates, Annu. Rev. Phys. Chem., 41, 527 (1990).

22. J. Trotter, Acta Crystallogr., 14, 1135 (1961).

23. P. Keller, B. Carvalho, J. P. Cotton, M. Lambert, F. Moussa, and G. Pepy, J. Phys. Lett., 46, L-1065 (1985).

24. P. Davidson and L. Strzelecki, Liq. Cryst., 3, 1583 (1988).

25. P. Davidson, A. M. Levelut, and M. F. Achard, Liq. Cryst., 4, 561 (1989).

26. A. J. Leadbetter and E. K. Norris, Mol. Phys., 38, 669 (1979).

27. A. B. Kunchenko and D. A. Svetogorsky, J. Physique, 47, 2015 (1986)

28. A. B. Kunchenko and D. A. Svetogorsky, Liq. Cryst., 2, 617 (1987).

29. P. Keller, B. Carvalho, J. P. Cotton, M. Lambert, F. Moussa, and G. Pepy, J. Phys. Lett., Paris, 46, L1065 (1985).

30. F. Moussa, J. P. Cotton, F. Hardouin, P. Keller, M. Lambert, G. Pepy, M. Mauzac, and H. Richard, J. Physique, 48, 1079 (1987).

31. L. Noirez, J.P. Cotton, F. Hardouin, P. Keller, F. Moussa, G Pepy, and C. Strazielle, Macromolecules, 21, 2889 (1988).

32. H. G. Ohm, R. G. Kirste, and R. C. Oberthur, Makromol. Chem., 116, 1378 (1988).

33. J. Kalus, S. G. Kostromin, V. P. Shibaev, A. B. Kunchenko, Yu. M. Ostanevich, and D. A. Svetogorsky, Mol. Cryst. Liq. Cryst., 155, 347 (1988)

34. P. Davidson, L. Noirez, J. P. Cotton, and P. Keller, Liq. Cryst., 10, 111 (1991).

35. C. Boeffel and H. M. Spiess, Macromolecules, 21, 1626 (1988).

36. M. D. Whitmore and J. Noolandi, Macromolecules, 21, 1482 (1988).

37. M. Gervais and B. Gallot, Polymer, 22, 1129 (1981).

38. K. C. Douzinas and R. E. Cohen, Macromolecules, 25, 5030 (1992).

39. R. Renz and M. Warner, Phys. Rev. Lett., 56, 1268 (1986).

40. J. Rieger, Mol. Cryst. Liq. Cryst., 155, 253 (1988).

41. J. Reiger, J. Phys. France, 49, 1615 (1988).

42. J. Reiger, J. Liq. Cryst., 5, 1559 (1989). 